\title{
ON SUBSPACES OF INVARIANT VECTORS
}

\author{
TATIANA SHULMAN
}

\begin{abstract}
Let $X_{\pi}$ be the subspace of fixed vectors for a uniformly bounded representation $\pi$ of a group $G$ on a Banach space $X$. We study the problem of the existence and uniqueness of a subspace $Y$ that complements $X_{\pi}$ in $X$. Similar questions for $G$ invariant complement to $X_{\pi}$ are considered. We prove that every non-amenable discrete group $G$ has a representation with non-complemented $X_{\pi}$ and find some conditions that provide an $G$-invariant complement. A special attention is given to representations on $C(K)$ that arise from an action of $G$ on a metric compact $K$.
\end{abstract}

\section{INTRODUCTION}

The subspaces of vectors which are invariant under group representations recently got new attention because of their use in the Banach space version of Kazhdan's property (T) (see [1], 6] ). In the Hilbert space case, arguments used for studying property (T) rely heavily on the existence of orthogonal complements of subspaces (of invariant vectors). In Banach space setting the lack of orthogonality immediately causes difficulties. It is not even clear if the subspace of invariant vectors is always complemented, as mentioned in [6]. However if a Banach space is superreflexive, then for any uniformly bounded representation, the subspace of invariant vectors is complemented ([1]). Moreover there is a complement which is invariant under the representation. Namely it is proved in [1] that if $\pi$ is a uniformly bounded representation of a group $G$ on a superreflexive space $X$, then $X$ decomposes into the sum

$$
X=X_{\pi} \oplus \operatorname{Ann}\left(X_{\bar{\pi}}^{*}\right),
$$

where $X_{\pi}$ is the subspace of invariant vectors, $X_{\bar{\pi}}^{*}$ is the subspace of vectors invariant under the dual representation $\bar{\pi}$ and $\operatorname{Ann}\left(X_{\bar{\pi}}^{*}\right)$ is its preannihilator. It is easy to check that $\operatorname{Ann}\left(X_{\bar{\pi}}^{*}\right)$ is $G$-invariant.

In this note we show that indeed the subspace of invariant vectors need not be complemented. Moreover, in section 1 we prove that each non-amenable group admits an isometric representation such that the subspace of invariant vectors is not complemented (Theorem 1).

In section 2 we study the decomposition $X=X_{\pi} \oplus A n n\left(X_{\bar{\pi}}^{*}\right)$, and more generally, the question of the existence and uniqueness of an invariant complement to $X_{\pi}$. By simple use of fixed point theorems we prove that the decomposition $X=X_{\pi} \oplus \operatorname{Ann}\left(X_{\bar{\pi}}^{*}\right)$ holds in some important cases, for instance for representations of compact groups (Theorem 5) and for uniformly bounded representations on reflexive Banach spaces (Theorem 4) which generalizes [[1], Prop. 2.6] since superreflexive spaces are reflexive. In these cases if the representation is isometric, then the corresponding projection onto $X_{\pi}$ has norm 1 . Though in general $G$-invariant complement need not be unique (Example 8), in the cases above it is unique. We show also that for any uniformly bounded representation of an

2000 Mathematics Subject Classification. 22A25; 46B99; 22D25.

Key words and phrases. uniformly bounded representations, continuous group actions, complemented subspaces, fixed point theorems. 
amenable group the subspaces $X_{\pi}$ and $\operatorname{Ann}\left(X_{\bar{\pi}}^{*}\right)$ have trivial intersection (Theorem 6). For non-amenable groups it is not true in general ([1], ex. 2.29).

In sections 3 and 4 we focus on representations coming from group actions on compact metric spaces. Though for such representations the decomposition $X=X_{\pi} \oplus \operatorname{Ann}\left(X_{\bar{\pi}}^{*}\right)$ need not hold in general, it is shown that it holds if the action is nice, namely Lyapunov stable (Theorem 15). Lyapunov stable actions were introduced in [5]. It was shown in [5] that if the action is Lyapunov stable, then there is a conditional expectation on the subspace (actually, subalgebra) of invariant functions. In Theorem 15 we give a new proof of that. Moreover we construct a conditional expectation commuting with the representation and show that such an expectation is unique. Along the way we give a short proof of an assertion in [5] on the uniqueness of invariant measures. In section 4 we introduce lower semi-continuous actions, which is a wider class of actions than Lyapunov stable ones. We show that for lower semi-continuous actions the subspace of invariant functions is complemented.

Acknowledgements. The author would like to thank Piotr Nowak for bringing the topic to her attention and for helpful discussions.

This research was partially supported by the Polish National Science Centre grant under the contract number DEC- 2012/06/A/ST1/00256.

\section{THE SUBSPACE OF INVARIANT VECTORS NEED NOT BE COMPLEMENTED}

Let $G$ be a topological group and $X$ be a Banach space. By a representation of $G$ on $X$ we will mean a strongly continuous homomorphism from $G$ into the group $B(X)$ of bounded invertible operators on $X$. A representation $\pi$ is isometric if $\pi(g)$ is an isometry for each $g \in G$ and $\pi$ is uniformly bounded if $\sup _{g \in G}\|\pi(g)\|<\infty$.

Below for any family $S \subseteq B(H)$ of operators on a Hilbert space by $S^{\prime}$ we denote its commutant, that is $S^{\prime}=\{T \in B(H) \mid T A=A T$, for any $A \in S\}$. Recall that the group von Neumann algebra $L(G)$ of a group $G$ is

$$
L(G)=\{\rho(g) \mid g \in G\}^{\prime \prime},
$$

where $\rho$ is the left regular representation of $G$.

Theorem 1. Any discrete non-amenable group admits an isometric representation such that the set of invariant vectors is not complemented.

Proof. Let $G$ be a non-amenable group, $H=l_{2}(G)$ and $\rho: G \rightarrow B(H)$ - the left regular representation. Define a representation $\tilde{\rho}: G \rightarrow B(H \otimes H)$ by

$$
\tilde{\rho}(g)=\rho(g) \otimes I d .
$$

Let $X=B(H \otimes H)$. Define a representation $\pi: G \rightarrow B(X)$ by

$$
\pi(g) x=\tilde{\rho}(g) x \tilde{\rho}(g)^{-1},
$$

$x \in X$. Let $N$ be the set of $\pi$-invariant vectors. Then

$$
\begin{gathered}
N=\left\{x \in X \mid \tilde{\rho}(g) x \tilde{\rho}(g)^{-1}=x, \forall g \in G\right\}= \\
\{\tilde{\rho}(g) \mid g \in G\}^{\prime}=\{\rho(g) \otimes I d \mid g \in G\}^{\prime}= \\
\quad\{T \otimes I d \mid T \in L(G)\}^{\prime}=(L(G) \otimes I d)^{\prime} .
\end{gathered}
$$

As is well known a von Neumann algebra is injective if and only if its commutant is injective ([3]). Since $G$ in non-amenable, $L(G)$ is non-injective ([2]). Since injectivity is 
preserved by $*$-isomorphisms, $L(G) \otimes I d$ is non-injective either and we conclude that $N$ is non-injective. Since

$$
(L(G) \otimes I d)^{\prime}=L(G)^{\prime} \otimes B(H),
$$

we have

$$
M_{2}(N) \cong N .
$$

By [[4], lemma 4.6], $N$ is not complemented in $X$.

Question: Does there exist a group which admits a uniformly bounded representation on a separable Banach space such that the set of invariant vectors is not complemented?

Question: Does there exist an amenable group which admits a uniformly bounded representation such that the set of invariant vectors is not complemented?

\section{On the DeComposition $X=X_{\pi} \oplus \operatorname{Ann}\left(X_{\bar{\pi}}^{*}\right)$}

For a representation $\pi$ one can define its adjoint representation $\bar{\pi}$ of $G$ on the dual space $X^{*}$ by

$x \in X, f \in X^{*}$.

$$
(\bar{\pi}(g) f)(x)=f\left(\pi\left(g^{-1}\right) x\right)
$$

For a subspace $Y \subseteq X$ by Ann $Y$ we will denote its annihilator in $X^{*}$, that is

$$
\text { Ann } Y=\left\{f \in X^{*} \mid f(x)=0 \text { for each } x \in Y\right\} \text {. }
$$

For a subspace $Y \subseteq X^{*}$ its preannihilator in $X$ we will denote also by $A n n Y$

$$
\text { Ann } Y=\{x \in X \mid f(x)=0 \text { for each } f \in Y\} \text {, }
$$

since it always will be clear from the context what we mean.

Let $X_{\pi}$ be the subspace of $\pi$-invariant vectors

$$
X_{\pi}=\{x \in X \mid \pi(g) x=x \text { for all } g \in G\} .
$$

Lemma 2. If $X_{\bar{\pi}}^{*}+\operatorname{Ann}\left(X_{\overline{\bar{\pi}}}^{* *}\right)$ is *-weakly dense in $X^{*}$, then $X_{\pi} \cap \operatorname{Ann}\left(X_{\bar{\pi}}^{*}\right)=\{0\}$.

Proof. It is easy to see that $X_{\pi} \cap \operatorname{Ann}\left(X_{\bar{\pi}}^{*}\right)$ annihilates the subspace $X_{\bar{\pi}}^{*}+\operatorname{Ann}\left(X_{\overline{\bar{\pi}}}^{* *}\right)$.

Lemma 3. Let $\pi$ be a representation of a group $G$ on a Banach space $X$.

(i) Suppose that for any $x \in X$, the closed convex hull $E(x)$ of its orbit $O(x)$ contains an invariant vector. Then

$$
X=X_{\pi}+\operatorname{Ann}\left(X_{\bar{\pi}}^{*}\right) .
$$

(ii) Suppose that for any $f \in X^{*}$, the $*$-weakly closed convex hull $E_{w}(f)$ of $O(f)$ contains an invariant vector. Then

$$
X^{*}=X_{\bar{\pi}}^{*}+\operatorname{Ann}\left(X_{\pi}\right) \text {. }
$$

Proof. (i) By the assumption, for $x \in X$, there is an invariant vector $x_{0} \in E(x)$. Let $f \in X_{\bar{\pi}}^{*}$. Then $f$ is constant on $O(x)$ and hence on $E(x)$. Hence $f\left(x-x_{0}\right)=0$. Thus $x-x_{0} \in \operatorname{Ann}\left(X_{\bar{\pi}}^{*}\right)$ and $X=X_{\pi}+\operatorname{Ann}\left(X_{\bar{\pi}}^{*}\right)$.

(ii) For $f \in X^{*}$, let $f_{0}$ be an invariant functional in $E_{w}(f)$. Let $x \in X_{\pi}$, then $f(x)=$ $f_{0}(x)$, and therefore $f-f_{0} \in \operatorname{Ann}\left(X_{\pi}\right), X^{*}=X_{\bar{\pi}}^{*}+\operatorname{Ann}\left(X_{\pi}\right)$.

Theorem 4. For any uniformly bounded representation $\pi$ on a reflexive Banach space $X$,

$$
X=X_{\pi} \oplus \operatorname{Ann}\left(X_{\bar{\pi}}^{*}\right) .
$$

The corresponding projection onto $X_{\pi}$ has norm less or equal to $\sup _{g \in G}\|\pi(g)\|$. 
Proof. Define an equivalent norm on $X$ by

$$
\|x\|^{\prime}=\sup _{g \in G}\|\pi(g) x\|
$$

With respect to this norm $\pi$ is isometric. So we can assume from the beginning that $\pi$ is an isometric representation on a reflexive space $X$.

Let $x \in X$. Let $O(x)$ and $E(x)$ be as in Lemma 3. Since $X$ is reflexive, $E(x)$ is a convex weakly compact invariant subset. By Ryll-Nardzewski theorem in $E(x)$ there is an invariant vector. By Lemma 3, $X=X_{\pi}+\operatorname{Ann}\left(X_{\bar{\pi}}^{*}\right)$. Since $X^{*}$ is also reflexive, we in the same way obtain $X^{*}=X_{\bar{\pi}}^{*}+\operatorname{Ann}\left(X_{\bar{\pi}}^{* *}\right)$. By Lemma $2, X=X_{\pi} \oplus \operatorname{Ann}\left(X_{\bar{\pi}}^{*}\right)$.

Let $P$ be the corresponding projection onto $X_{\pi}$. By the proof of Lemma 3 , for any $x \in X$, $P x$ belongs to the closed convex hull of the orbit of $x$. Hence $\|P x\| \leq \sup _{g \in G}\|\pi(g) x\|$, for any $x \in X$, and $\|P\| \leq \sup _{g \in G}\|\pi(g)\|$.

Theorem 5. For any representation $\pi$ of a compact group on a Banach space $X$,

$$
X=X_{\pi} \oplus \operatorname{Ann}\left(X_{\bar{\pi}}^{*}\right) .
$$

If $\pi$ is isometric, then the corresponding projection onto $X_{\pi}$ has norm 1.

Proof. Let $x \in X$. Let $O(x)$ and $E(x)$ be as in Lemma 3. Since the group is compact, $O(x)$ is compact. Hence $E(x)$ is a convex compact invariant subset. By Kakutani's fixed point theorem in $E(x)$ there is an invariant vector. By Lemma $3 X=X_{\pi}+\operatorname{Ann}\left(X_{\bar{\pi}}^{*}\right)$. The same applies to $\bar{\pi}$, so we also get $X^{*}=X_{\bar{\pi}}^{*}+\operatorname{Ann}\left(X_{\overline{\bar{\pi}}}^{* *}\right)$. By Lemma 2 ,

$$
X=X_{\pi} \oplus \operatorname{Ann}\left(X_{\bar{\pi}}^{*}\right) .
$$

The same argument as in Theorem 4 shows that the projection onto $X_{\pi}$ has norm 1.

Theorem 6. If $\pi$ is a uniformly bounded representation of an amenable group on a Banach space $X$ then

(i) $X^{*}=X_{\bar{\pi}}^{*}+\operatorname{Ann}\left(X_{\pi}\right)$,

(ii) $X_{\pi} \cap \operatorname{Ann}\left(X_{\bar{\pi}}^{*}\right)=\{0\}$.

Proof. (i) For each $f \in X^{*}$, the $*$-weakly closed convex span $E_{w}(f)$ of $O(f)$ is $*$-weakly compact. Since all the operators $\bar{\pi}(g), g \in G$, are $*$-weakly continuous, it follows from amenability that $E_{w}(f)$ contains a fixed point of $\bar{\pi}$. Now by Lemma 2 we conclude that $X^{*}=X_{\bar{\pi}}^{*}+\operatorname{Ann}\left(X_{\pi}\right)$.

(ii) It is easy to see that $X_{\bar{\pi}}^{*}+\operatorname{Ann}\left(X_{\pi}\right)$ annihilates $X_{\pi} \cap \operatorname{Ann}\left(X_{\bar{\pi}}^{*}\right)$.

Note that the decomposition in (i) is not necessarily direct. For example if $X=l^{1}(\mathbb{Z})$, $G=\mathbb{Z}$ and $\pi(n) f(k)=f(n+k)$ then $X_{\pi}=0, \operatorname{Ann}\left(X_{\pi}\right)=X^{*}, X_{\bar{\pi}}^{*}$ is the space of constant sequences. Similarly in this example $X \neq X_{\pi}+\operatorname{Ann}\left(X_{\bar{\pi}}^{*}\right)$.

Proposition 7. If $X_{\pi}$ has a $\pi(G)$-invariant complement $Y$, then $Y \supseteq \operatorname{Ann}\left(X_{\bar{\pi}}^{*}\right)$. In particular, in Theorems 4 and 5 the space $X_{\pi}$ has unique $\pi(G)$-invariant complement.

Proof. Let $P$ be the projection onto $X_{\pi}$ parallel to $Y$. Since $Y$ is $\pi(G)$-invariant, $[P, \pi(g)]=$ 0 , for all $g \in G$. Hence for any $x \in X$

$$
P(\pi(g) x-x)=\pi(g) P x-P x=0 .
$$

Thus $\pi(g) x-x \in Y$. Let $f \in X^{*}$ and $\left.f\right|_{Y}=0$. Then $f(\pi(g) x-x)=0$, for all $g \in G$, $x \in X$, that is $f \in X_{\bar{\pi}}^{*}$. Hence $Y^{\perp} \subseteq X_{\bar{\pi}}^{*}$, whence $Y \supseteq \operatorname{Ann}\left(X_{\bar{\pi}}^{*}\right)$.

In general a $\pi(G)$-invariant complement need not be unique as shows Example 8 below. 


\section{LyAPUNOV STABLE ACTIONS}

Let $K$ be a metric compact space and let group $G$ act continuously on $K$. Define a representation $\pi$ of $G$ on $C(K)$ by

$$
\pi(g) \phi(x)=\phi\left(g^{-1} x\right) .
$$

The following example shows that the decomposition

$$
C(K)=C(K)_{\pi} \oplus \operatorname{Ann}\left(C(K)_{\bar{\pi}}^{*}\right)
$$

does not hold in general, even when the group is abelian. It also shows that $\pi(G)$-invariant complement need not be unique.

Example 8. Let $K=[0,1]$ and let $G$ be the group $\mathbb{Q}_{+}=\left\{\frac{m}{n} \mid m, n \in \mathbb{N}\right\}$ with the usual multiplication and the discrete topology. Define a continuous action $\alpha$ of $G$ on $K$ as

$$
\alpha\left(\frac{m}{n}\right) x=x^{\frac{m}{n}} .
$$

Define a representation $\pi$ of $G$ on $C(K)$ by

$$
\pi(g) \phi(x)=\phi\left(\alpha\left(g^{-1}\right) x\right) .
$$

Let us show that $C(K)_{\pi}$ is the subspace of constant functions. For each $x \in[0,1)$, $0 \in \overline{O(x)}$. Hence for $\phi \in C(K)_{\pi}$ and each $x \in[0,1), \phi(x)=\phi(0)$. Thus $\phi=$ const. We will show now that $\operatorname{Ann}\left((C(K))_{\bar{\pi}}^{*}\right) \subseteq C_{0}(0,1)$. Define $h_{i} \in C(K)^{*}, i=1,2$, as $h_{1}(\phi)=\phi(0), h_{2}(\phi)=\phi(1)$, for any $\phi \in C(K)$. It is easy to see that $h_{i}, i=1,2$, are constant on orbits of functions in $C(K)$ and hence $h_{i} \in(C(K))_{\bar{\pi}}^{*}, i=1,2$. Hence

$$
\operatorname{Ann}\left((C(K))_{\bar{\pi}}^{*}\right) \subseteq \operatorname{Ann}\left(h_{1}\right) \cap \operatorname{Ann}\left(h_{2}\right)=C_{0}(0,1] \cap C_{0}[0,1)=C_{0}(0,1) .
$$

Thus Ann $\left((C(K))_{\bar{\pi}}^{*}\right)$ does not complement $C(K)_{\pi}$. However $C(K)_{\pi}$ has $\pi(G)$-invariant complements $C_{0}(0,1]=\{\phi \in C(K) \mid \phi(0)=0\}$ and $C_{0}[0,1)=\{\phi \in C(K) \mid \phi(1)=0\}$ (and many others).

However we will show that if an action is nice enough (namely, Lyapunov stable) then the decomposition

$$
C(K)=C(K)_{\pi} \oplus \operatorname{Ann}\left(C(K)_{\bar{\pi}}^{*}\right)
$$

holds.

Definition 9. An action of $G$ on $K$ is Lyapunov stable if for any $\epsilon>0$ there is $\delta=$ $\delta(\varepsilon)>0$ such that $d(g x, g y)<\varepsilon$, for all $g \in G$, whenever $d(x, y)<\delta$.

Remark 10. The original definition in [5] was different: for any $x \in K$ and $\epsilon>0$ there must exist $\delta=\delta(x, \varepsilon)>0$ such that $d(g x, g y)<\varepsilon$, for all $g \in G$, whenever $d(x, y)<\delta$. But a standard compactness argument shows that for compact $K$ the definitions coincide. Indeed for each $x \in K$, let $U_{x}=\{y \in K: d(y, x)<\delta(x, \varepsilon / 2) / 2\}$ and choose a finite subcovering $U_{x_{1}}, \ldots, U_{x_{n}}$. Let $\delta=\min _{i} \delta\left(x_{i}, \varepsilon / 2\right) / 2, i=1, \ldots, n$. If $d(x, y) \leq \delta$, one can find $i$ with $x \in U_{x_{i}}$, then $d\left(y, x_{i}\right) \leq \delta+\delta\left(x_{i}, \varepsilon / 2\right) / 2 \leq \delta\left(x_{i}, \varepsilon / 2\right)$. It follows that $d\left(g x, g x_{i}\right) \leq \varepsilon / 2$ and $d\left(g y, g x_{i}\right) \leq \varepsilon / 2$ whence $d(g x, g y) \leq \varepsilon$.

Below by $\pi$ we always mean the representation coming from some group action on a compact.

Lemma 11. Let an action of $G$ on $K$ be Lyapunov stable and $\pi$ be as above. Then for any $\phi \in C(K)$, its orbit $O(\phi)$ is precompact. 
Proof. It is easy to see that Lyapunov stability implies that for any $\phi \in C(K), O(\phi)$ is an equicontinuous family of functions. Since $O(\phi)$ is bounded, it is precompact by Arzela - Ascoli theorem.

Lemma 12. Let an action of $G$ on $K$ be Lyapunov stable and $\pi$ be as above. Then

$$
C(K)=C(K)_{\pi}+\operatorname{Ann}\left(C(K)_{\bar{\pi}}^{*}\right) \text {. }
$$

Proof. It follows from Lemma 11, that for any $\phi \in C(K)$, the closed convex hull $E(\phi)$ of the orbit $O(\phi)$ is compact. By Kakutani's fixed point theorem $E(\phi)$ contains a common fixed point for all $\pi(g)$, that is an invariant vector. By Lemma 3 ,

$$
C(K)=C(K)_{\pi}+\operatorname{Ann}\left(C(K)_{\bar{\pi}}^{*}\right) .
$$

Now we obtain a short proof of an assertion in [5] on the uniqueness of invariant measures.

Corollary 13. ([5], Lemma 6.1) Suppose a group $G$ acts on a compact metric space $K$ in such a way that the orbit of an element $a \in K$ is dense in $K$. If the action is Lyapunov stable, then $K$ carries not more than one invariant regular measure.

Proof. By Lemma 12, $C(K)=C(K)_{\pi}+\operatorname{Ann}\left(C(K)_{\bar{\pi}}^{*}\right)$. Since the orbit of $a \in K$ is dense in $K$, the only invariant functions are constants, so $C(K)_{\pi}=\mathbb{C}$. Hence $\operatorname{codim} \operatorname{Ann}\left(C(K)_{\bar{\pi}}^{*}\right) \leq$ 1 and $\operatorname{dim} C(K)_{\bar{\pi}}^{*} \leq 1$. The latter exactly means that there is not more than one invariant regular measure on $K$, because regular measures are in one-to one correspondence with points of $C(K)^{*}$ by Riesz theorem.

Lemma 14. Let $\pi$ be a representation of a group $G$ on a Banach space $X$ such that all orbits are precompact. Let $E \subset X^{*}$ be a bounded $\bar{\pi}(G)$-invariant subset. Then the group of maps $\left\{\left.\bar{\pi}(g)\right|_{E}\right\}_{g \in G}$ is equicontinuous with respect to the relative *-weak topology on $E$.

Proof. Let $\epsilon>0$ and $x_{1}, \ldots, x_{N} \in X$. We need to find $\delta>0$ and $y_{1}, \ldots, y_{m} \in X$ such that

$$
\left|\left(\bar{\pi}(g)\left(h_{1}-h_{2}\right)\right)\left(x_{i}\right)\right|<\epsilon, g \in G, i=1, \ldots, N
$$

whenever $h_{1}, h_{2} \in E$ and $\left|h_{1}\left(y_{j}\right)-h_{2}\left(y_{j}\right)\right|<\delta, j=1, \ldots, m$. Let $M=\sup _{h \in E}\|h\|$. As $\left\{y_{j}\right\}$ we take a $\frac{\epsilon}{6 M}$-net in $\bigcup_{i=1}^{N} \overline{O\left(x_{i}\right)}$ which exists since all orbits are precompact. Let $\delta=\epsilon / 3$. Then for any $h_{1}, h_{2} \in E$ such that $\left|h_{1}\left(y_{j}\right)-h_{2}\left(y_{j}\right)\right|<\delta, j=1, \ldots, m$, and for any $g \in G$ we have

$\left|\left(\bar{\pi}(g)\left(h_{1}-h_{2}\right)\right)\left(x_{i}\right)\right|=\left|\left(h_{1}-h_{2}\right)\left(\pi\left(g^{-1}\right) x_{i}\right)\right| \leq\left|\left(h_{1}-h_{2}\right)\left(\pi\left(g^{-1}\right) x_{i}-y_{k}\right)\right|+\left|\left(h_{1}-h_{2}\right)(y)\right|$

(where $k=k(i, g)$ is chosen in such a way that $\left\|\pi\left(g^{-1}\right) x_{i}-y_{k}\right\| \leq \frac{\epsilon}{6 M}$ )

$$
\leq\left\|h_{1}-h_{2}\right\| \frac{\epsilon}{6 M}+\epsilon / 3<\epsilon / 3+\epsilon / 3 \leq \epsilon
$$

Theorem 15. Let an action of $G$ on $K$ be Lyapunov stable and $\pi$ be as above. Then

$$
C(K)=C(K)_{\pi} \oplus \operatorname{Ann}\left(C(K)_{\bar{\pi}}^{*}\right) .
$$

The corresponding projection onto $C(K)_{\pi}$ is a conditional expectation.

Proof. Let $f \in C(K)^{*}$ and $E(f)$ be the closed convex hull of $O(f)=\{\bar{\pi}(g) f\}_{g \in G}$. Then $E(f)$ is a convex *-weakly compact $\bar{\pi}(G)$-invariant subset. By Lemma 14 and Kakutani's fixed point theorem, in $E(f)$ there is a $\bar{\pi}(G)$-invariant vector. By Lemma 3

$$
C(K)^{*}=C(K)_{\bar{\pi}}^{*}+\operatorname{Ann}\left(C(K)_{\overline{\bar{\pi}}}^{* *}\right) .
$$


The decomposition $C(K)=C(K)_{\pi} \oplus \operatorname{Ann}\left(C(K)_{\bar{\pi}}^{*}\right)$ follows now from Lemma 2, Lemma 12 and (31). The same argument as in Theorem 4 shows that the projection onto $X_{\pi}$ has norm 1. Since $C(K)$ and $C(K)_{\pi}$ are $C^{*}$-algebras, by [7] it is a conditional expectation.

\section{LOWER SEMI-CONTINUOUS ACTIONS}

Now we will show that for more general actions than Lyapunov stable, namely for lower semi-continuous actions, the subspace $C(K)_{\pi}$ is complemented.

Let $X$ be a compact metric space. Let $M$ be a partition of $X$ into closed subsets. For $x \in X$ let $M(x)$ denote the member of $M$ which contains $x$. Corresponding to the standard definitions $M$ is called lower semi-continuous if $\{x \in X: M(x) \bigcap U \neq \emptyset\}$ is an open set in $X$ for every open set $U$ in $X$.

If $P \subseteq C(X)$ is a subspace, then the $P$-partition of $X$ is the partition associated with the following equivalence relation $\mathrm{R}$. A couple $(x, y) \in R$ if and only if $p(x)=p(y)$ for every $p \in P$. Now let

$$
K(P)=\bigcup\{K \subseteq X: K \text { is a member of the P-partition of } \mathrm{X},
$$

$$
\text { and } \mathrm{K} \text { contains more than one point of } X\}
$$

According to [8], $P$ has a lower semi-continuous quotient if the restriction of the $P$ partition to $\overline{K(P)}$ is lower semi-continuous.

Definition 16. We will say that an action is lower semi-continuous if the corresponding $C(K)_{\pi}$-partition is lower semi-continuous.

We don't know any example of a not lower semi-continuous action. An easy example of a lower semi-continuous action is the action from Example 8. Other examples are Lyapunov stable actions as we show below.

Lemma 17. For any continuous action and for any open $U \subseteq K$, the set $\{x \mid O(x) \cap U \neq$ $\emptyset\}$ is open.

Proof. Let $E=\{x \mid \overline{O(x)} \cap U \neq \emptyset\}$. Since $U$ is open, $x \in E$ implies that there is $g \in G$ and $\epsilon>0$ such that the ball $B_{\epsilon}(g x)$ is inside $U$. There is $\delta$ such that $d(g x, g y) \leq \epsilon$ whenever $d(x, y)<\delta$. Thus for any $y \in B_{\delta}(x)$ we have $\left.g y \in B_{\epsilon}(g x)\right) \subset U$. Hence $y \in E$. Thus $E$ is open.

We are going to use the following result from [5].

Theorem 18. ([5], Lemma 3.1) For a Lyapunov stable action, any two orbits are either separated from each other, or have the same closure. The quotient space of closures of orbits is Hausdorff.

The following corollary shows that for Lyapunov stable actions the partition into closed orbits and the $C(K)_{\pi}$-partition are the same.

Corollary 19. Suppose we have a Lyapunov stable action and let $R$ be the equivalence relation defining $C(K)_{\pi}$-decomposition of $K$. Then $(x, y) \in R$ if and only if $\overline{O(x)}=\overline{O(y)}$.

Proof. Since functions in $C(K)_{\pi}$ are those which are constant on orbits, the "if" part follows.

To prove the "only if" part, assume that $\overline{O(x)} \neq \overline{O(y)}$. Then by Urysohn's lemma and Theorem 18, there is a continuous function $\psi$ on $K / s$ such that $\psi(\overline{O(x)}) \neq \psi(\overline{O(y)})$. Define $\phi \in C(K)$ by $\phi(x)=\psi(\overline{O(x)})$. Then $\phi \in C(K)_{\pi}$ and $\phi(x) \neq \phi(y)$, hence $(x, y) \notin R$. 
Theorem 20. Lyapunov stable actions are lower semi-continuous.

Proof. For any open $U \subseteq K$ we need to check that the set

$$
E=\{x \in K \mid R(x, u)=0 \text {, for some } u \in U\}
$$

is open. By Corollary 19 and Theorem 18

$$
E=\{x \mid \overline{O(x)}=\overline{O(u)} \text {, for some } u \in U\}=\{x \mid \overline{O(x)} \cap U \neq \emptyset\}=\{x \mid O(x) \cap U \neq \emptyset\}
$$

which is open by Lemma 17 .

Now we will show that for lower semi-continuous actions, the subspace $C(K)_{\pi}$ is always complemented.

Lemma 21. Let $P \subseteq C(X)$ be a subspace such that $P$-partition of $X$ is lower semicontinuous. Then $P$ has lower semi-continuous quotient.

Proof. It suffices to show that a subpartition of a lower semi-continuous partition is lower semi-continuous. Let a partition $M$ be lower semi-continuous and $M_{0}$ be its subpartition. Let $K_{0}$ be the closure of the union of members of $M_{0}$. Suppose that $U$ is open in $K_{0}$. We need to show that $\left\{x \in K_{0} \mid M_{0}(x) \bigcap U \neq \emptyset\right\}$ is open in $K_{0}$. Since $U \bigcup\left\{K \backslash K_{0}\right\}$ is open in $K$ (because its complement is $K_{0} \backslash U$ ) and $M$ is lower semi-continuous, the set

$$
\left\{x \in K \mid M(x) \bigcap\left(U \bigcup\left\{K \backslash K_{0}\right\}\right) \neq \emptyset\right\}
$$

is open, whence

$$
\left\{x \in K_{0} \mid M_{0}(x) \bigcap U \neq \emptyset\right\}=\left\{x \in K \mid M(x) \bigcap\left(U \bigcup\left\{K \backslash K_{0}\right\}\right) \neq \emptyset\right\} \bigcap K_{0}
$$

is open in $K_{0}$.

Proposition 22. Suppose $G$-action on $K$ is lower semi-continuous. Then $C(K)_{\pi}$ is complemented.

Proof. Obviously $C(K)_{\pi}$ is a $C^{*}$-subalgebra of $C(K)$ and hence is isomorphic to $C(Z)$, for some Hausdorff space $Z$. The statement follows now from Lemma 21 and [Th. 4, [8]].

\section{REFERENCES}

[1] U. Bader, A. Furman, T. Gelander, N. Monod, Property (T) and rigidity for actions on Banach spaces, Acta Mathematica 198 (2007), no. 1, 57 - 105.

[2] N. P. Brown and N. Ozawa, $C^{*}$-algebras and finite-dimensional approximations, Graduate Studies in Mathematics, vol. 88, American Mathematical Society, Providence, RI, 2008.

[3] A. Connes, Noncommutative geometry, Academic Press, San Diego, CA, 1994.

[4] U. Haagerup, G. Pisier, Bounded linear operators between $C^{*}$-algebras, Duke Math. Journ., 71(1993), $889-925$.

[5] M. Frank, V. Manuilov, E. Troitsky, Hilbert $C^{*}$-modules from group actions: beyond the finite orbit case, Studia Math. 200 (2010), no. 2, 131 - 148.

[6] P. W. Nowak, Group actions on Banach spaces, Handbook of group actions. Vol. II, Adv. Lect. Math. (ALM), vol.32, Int. Press, Somerville, MA, 2015, pp. 121 - 149.

[7] J. Tomiyama, On the projection of norm one in $W^{*}$-algebras, Proc. Japan Acad. 33 (1957), 608 612.

[8] D. E. Wulbert, Some complemented function spaces in $C(X)$, Pacific J. Math., Vol. 24, 3, 1968.

INSTITUTE OF MATHEMATICS OF THE POLISH ACADEMY OF SCIENCES, POLAND

E-mail address: tshulman@impan.pl 